\title{
The application of BP neural network in the safety warning of gas stations
}

\author{
Li Qiao $^{1, a^{*}}$, Jin Yüb \\ ${ }^{1}$ 10Xitu Road,Haidian District,Beijing,China \\ ${ }^{2} 10$ Xitu Road,Haidian District,Beijing,China \\ aqiaolifly@163.com, byujin_bupt@sina.com
}

Keywords: BP neural network, gas filling station, safety warning.

Abstract. In this paper, we analyze the current situation of gas filling station, find out the risk factors affecting the safety of gas stations, and propose a new algorithm based on BP neural network model. With the historical monitoring data of gas filling stations, BP neural network model can be used to approximate any nonlinear continuous function, and the historical data of each index are studied. The data were randomly divided into training data and test data. Reasonable selection of network layer number and node number, a large number of experiments were carried out, and the hidden rule is established. The risk prediction model based on BP neural network system was established. The accuracy of the model was verified by Matlab simulation. This study is conducive to improve the safety performance of the filling station, and find out the potential danger in advance, improve the efficiency of accident rescue, and play an important role in reducing the accident loss.

\section{Introduction}

$\mathrm{CNG}($ Compressed Natural Gas)is a low cost, high efficiency and less pollution of natural gas pressure, which is stored in the container in the form of gas, and has a broad application prospect in the field of energy substitution [1]. Gas filling stations which is a place with a large number of CNG storage, is the focus of the security monitoring department. Due to the large amount of flammable and explosive material stored in the gas filling station, and the operation is frequent, the accident can cause significant property damage, so it has important significance to analyze the historical monitoring data, find out the key monitoring targets and divide and predict the risk level[2].

There are many factors that affect the safety index in the gas station. Because of the large amount of data is nonlinear, time and other reasons, it is difficult to find the mathematical model which can accurately describe the data variable and dangerous level. Neural network system has the characteristics of adaptive learning, nonlinear approximation, reasoning ability and associative memory ability, and has wide application in signal processing, control and nonlinear fitting[3]. It provides powerful tool for solving complex nonlinear problems. Therefore, the Matlab neural network in BP can be used to design a reasonable model for the safety monitoring data.

\section{BP neural network}

BP neural network which is proposed by Rumelhart and McCelland in 1989 is a multilayer feedforward network by error back propagation training algorithm. It has broad application in predicting accidents and safety evaluation with good scene reappearance ability [4].

In the neural network system, there is no feedback connection between the neurons, but by the output error of the back propagation. It modifies the network weights layer by layer to make network error square sum minimum. So we achieve the purpose of accurate system output [5].

BP neural network structure. The topology structure of BP neural network model includes input layer, hidden layer and output layer. It may contain one or more hidden nodes. Data input from the input layer node, 
through the hidden layer nodes, finally outputting. Among them, there is no coupling relationship between the nodes of the same layer and each layer of the node only through the weight to affect the next layer node [6]. Model structure diagram is shown in figure 1.

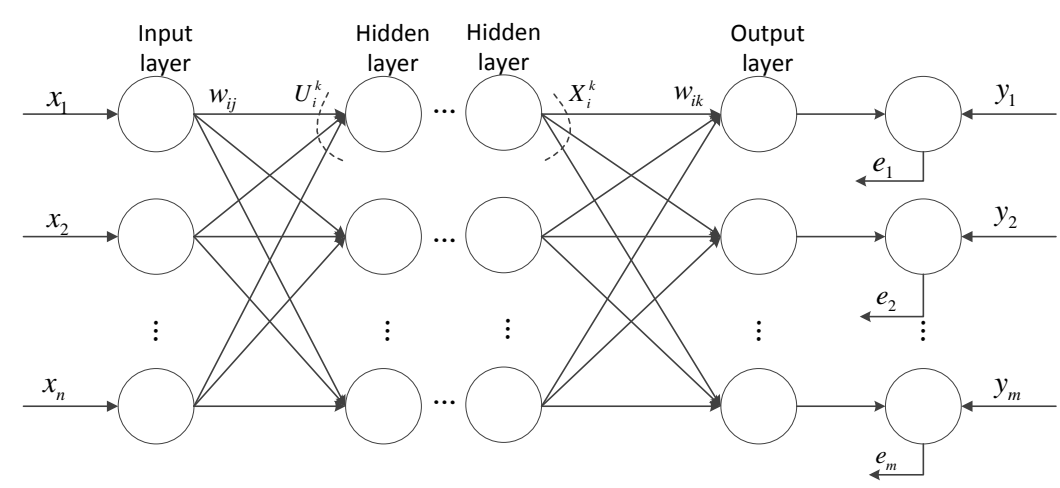

Fig. 1 Model structure diagram

BP neural network algorithm. BP neural network algorithm is proposed based on the existing algorithm of neural network. The given target output is directly used as a linear equation set, which is based on a set of weights. The BP neural network algorithm has no local minimum and slow convergence contrasting to the traditional method [7].

BP algorithm is a gradient search traversal network based on the Delta learning rule, so that the error between the desired output results and the actual results will be the smallest [8]. The basic ideas of the BP neural network algorithm are described in the following ways.

The number of BP neural network layers is $\mathrm{m}$, and the input layer neuron is $\mathrm{X}$, the output layer neuron is Y. $U_{i}^{k}$ is the total input of the I neuron of the k layer, and $X_{i}^{k}$ is the total output of the neuron. $f$ is excitation function. So we can get the following result:

$$
X_{i}^{k}=f\left(U_{i}^{k}\right)
$$

$W_{i j}$ is the weights between the $\mathrm{j}$ neuron of the $\mathrm{k}-1$ layer and the i neuron of layer $\mathrm{k}$, and we can get the following result:

$$
U_{i}^{k}=\sum_{j} W_{i j} X_{j}^{k-1}
$$

The definition of $Y_{i}$ is the output of the expected value, and $X_{i}{ }^{m}$ is the actual output. The error function is the square sum of the difference between the expected output and the actual output, and the error function $e$ is:

$$
e=\frac{1}{2} \sum_{i}\left(X_{i}^{m}-Y_{i}\right)^{2}
$$

Therefore, the weight of the change $\Delta w_{i j}$ is:

$$
\Delta W_{i j}=-\frac{\partial e}{\partial W_{i j}}=-\frac{\partial e}{\partial U_{i}^{k}} X_{j}^{k-1}
$$

We can get the following result through further derivation:

$$
\frac{\partial e}{\partial U_{i}^{k}}=X_{i}^{k}\left(1-X_{i}^{k}\right) \cdot \sum_{i} W_{i}^{j} \cdot \frac{\partial e}{\partial U_{i}^{k+1}}
$$

From the above deduction process, the BP neural network training method is that input the training sample to the input layer, output to the output layer through the transfer function, and output finally. We compare the 
final output with the actual output so that we can get the error signal. The weight value is modified according to the formula to make the error minimum [9].

\section{Safety warning model of gas filling station based on BP neural network}

Model overvie w. Due to the daily monitoring of gas filling stations is heavy and there is not a full range of early warning strategy, most stations give an alarm by setting up dangerous gas sensors in the plants. But this warning mechanism can only be detected in little time before the risk. So we study a kind of early warning model to predict the unknown risk which is useful to maintain the safety of gas stations operation and reduce the risk of accidents.

According to the existing data of gas station, we select the sample data and process the data, and then carry out the training of the model. Among them, the neural network model flow chart is shown in figure 2.

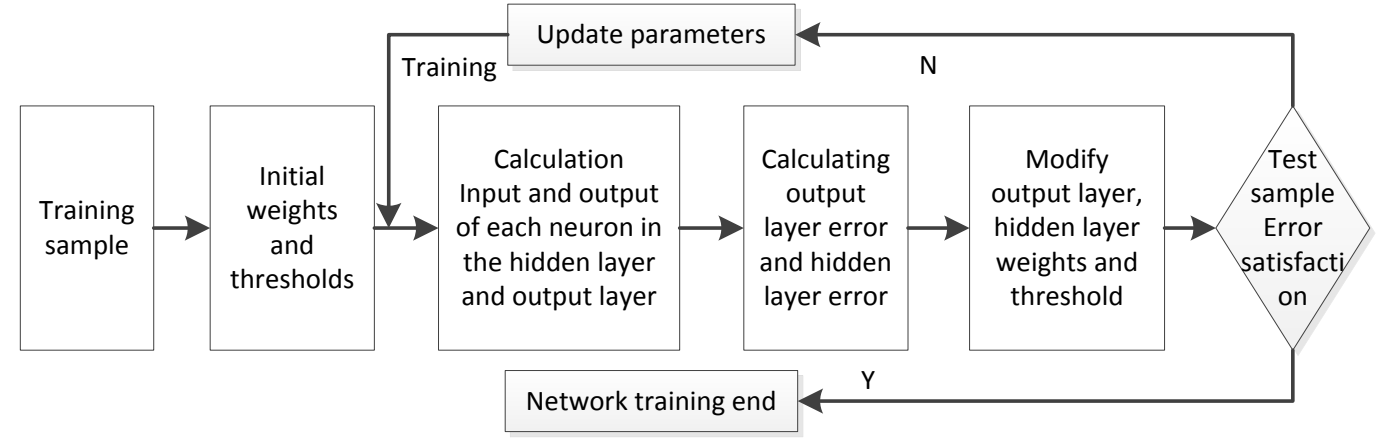

Fig. 2 The neural network model flow chart

The key of using the BP neural network model to predict the security is to design the network topology. Usually, the following contents are included: determining the input and output nodes, the number of neurons in the BP neural network model, the number of hidden neurons, and the determination of the neuron excitation function [10].

Model establishment. First we choose the input and output nodes. Due to the large number of sensors to monitor the gas station and the data number is also great, so it is particularly important to select the appropriate object and get the appropriate data. By taking into account that the monitoring points and the data have a certain degree of volatility, we select the historical monitoring data which include level one warning, level two warning, and level three warning. We choose one hundred sets of data from every level warning. So we get three hundred sets of characteristic signal which are six dimensional signals as inputs. The signal characteristics were Storage tank pressure, the temperature difference at room temperature and tank, unit volume LNG and oxygen ratio in the air, cylinder using year number, daily ventilation frequency, $\mathrm{H}_{2} \mathrm{~S}$ content in LNG and score. The alert level in the data is converted to a digital output by the score standard. Part of the data is shown in the table 1.

Because the alarm level of the input signal has 6 dimensions, so the input layer has 6 nodes, the output layer has 1 node. 
Table 1.Part of the data

\begin{tabular}{|c|c|c|c|c|c|c|}
\hline $\begin{array}{l}\text { Storage } \\
\text { tank } \\
\text { pressure } \\
{[\mathrm{Mpa}]} \\
\end{array}$ & $\begin{array}{l}\text { The temperature } \\
\text { difference at room } \\
\text { temperature and } \\
\text { tank }\left[{ }^{\circ} \mathrm{C}\right]\end{array}$ & $\begin{array}{l}\text { Unit volume } \\
\text { LNG and } \\
\text { oxygen ratio in } \\
\text { the air[\%] }\end{array}$ & $\begin{array}{l}\text { Cylinder } \\
\text { using year } \\
\text { number } \\
\text { [year] }\end{array}$ & $\begin{array}{l}\text { Daily } \\
\text { ventilation } \\
\text { frequency } \\
\text { [time] } \\
\end{array}$ & $\begin{array}{l}\mathrm{H}_{2} \mathrm{~S} \\
\text { content in } \\
\mathrm{LNG} \\
{\left[\mathrm{mg} / \mathrm{m}^{3]}\right.}\end{array}$ & Score \\
\hline 0.81 & 10 & 0.08 & 1 & 1 & 0.18 & 30 \\
\hline 0.71 & 12 & 0.08 & 3 & 1 & 0.21 & 26 \\
\hline 0.87 & 8 & 0.09 & 5 & 2 & 0.22 & 11 \\
\hline 0.32 & 9 & 0.12 & 3 & 0 & 0.23 & 29 \\
\hline 0.79 & 8 & 0.04 & 2 & 1 & 0.22 & 26 \\
\hline 0.85 & 9 & 0.03 & 2 & 0 & 0.22 & 3 \\
\hline 0.91 & 11 & 0.06 & 5 & 1 & 0.21 & 19 \\
\hline 0.91 & 9 & 0.07 & 4 & 2 & 0.23 & 6 \\
\hline 0.93 & 9 & 0.08 & 5 & 1 & 0.21 & 24 \\
\hline 0.82 & 10 & 0.09 & 4 & 1 & 0.22 & 17 \\
\hline 0.75 & 11 & 0.09 & 3 & 1 & 0.24 & 12 \\
\hline 0.89 & 4 & 0.06 & 5 & 1 & 0.21 & 14 \\
\hline 0.74 & 7 & 0.08 & 3 & 1 & 0.23 & 32 \\
\hline 0.82 & 8 & 0.08 & 5 & 1 & 0.21 & 13 \\
\hline 0.71 & 6 & 0.11 & 5 & 2 & 0.23 & 17 \\
\hline 0.66 & 12 & 0.12 & 4 & 2 & 0.23 & 25 \\
\hline 0.84 & 11 & 0.11 & 5 & 0 & 0.23 & 26 \\
\hline 0.65 & 10 & 0.09 & 5 & 1 & 0.25 & 11 \\
\hline 0.71 & 11 & 0.10 & 3 & 0 & 0.21 & 20 \\
\hline 0.88 & 12 & 0.22 & 3 & 1 & 0.18 & 29 \\
\hline 0.88 & 12 & 0.11 & 5 & 0 & 0.17 & 14 \\
\hline
\end{tabular}

Second we determinate the number of layers. Usually, the output error can be reduced by increasing the number of network layers, but at the same time, the network will become complex and the response speed will be slow. For gas filling station, there is no complicated input and output relationship, so using the three layer BP neural network structure can meet the system requirements. For the predict data in this paper, we determine the BP network structure for the three layers which is the input layer, one layer of hidden layer and the output layer.

Third we choose the number of hidden neurons. It should be able to reflect the input and output relationship correctly, and then try to get the fewer number to make the network simple. Therefore, we use the following empirical formula to determine the number of hidden layer neurons:

$$
\mathrm{j}=\sqrt{\mathrm{n}+\mathrm{m}}+\alpha
$$

Among them, $\mathrm{n}$ is the number of nodes in the input layer, mis the number of nodes in the output layer, $\alpha$ is a constant from 1-10 [11]. In this paper, the number of hidden layers is determined as 8 .

The last one is the selection of neuron excitation function. In the neuron model, the function of the excitation function can be used in many different forms. We commonly use the threshold function, the piecewise linear function and the Sigmoid function [12].

Since the Sigmoid function can be used to transform the input which is in positive and negative infinity into the output which is in -1 to 1 . So the Sigmoid function is chosen as the excitation function. Sigmoid function is as follows: 
$f(x)=\frac{1}{1+e^{-x}}$

\section{MATLAB implementation and results analysis}

Model simulation. We use the neural network toolbox in Matlab to simulation experiments. The main steps are as follows:

(1) 300 sets of data are randomly divided into 200 sets training data and 100 sets of forecast data. The main code is as follows:

Input_train=input (n (1:200),:)';

Output_train=output (n $(1: 200))^{\prime}$;

Input_test=input (n (201:300),:)';

Output_test=output (n (201:300))';

Input_train is the input training data, output_train is the output training data, input_test is the input forecast data and output_test is the output forecast data.

(2) We use Matlab own function mapminmax to do normalized treatment to make data in the $(-1,1)$ range. The code is as follows:

[inputn, inputps]=mapminmax (input_train);

[outputn, outputps]=mapminmax (output_train);

(3)We write the following code to create the BP network: net=newff (inputn, outputn, 8);

(4) The BP neural network is trained and the code is as follows:

Net.trainParam.epochs $=100$;

Net.trainParam.lr $=0.1$;

Net.trainParam.goal=0.00004;

Net=train (net, inputn, outputn);

Result analysis. After 100 iterations of the model, the output results are shown in figure 3 and figure 4 . Figure 3 is the contrast between the expected output and the expected output, figure 4 is the percentage of the forecast error.

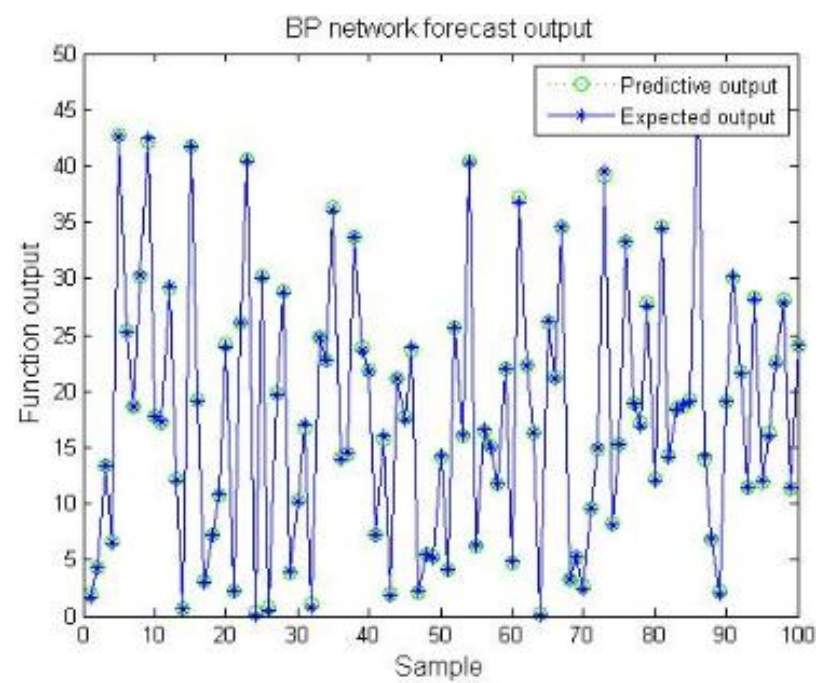

Fig. 3 BP network forecast output

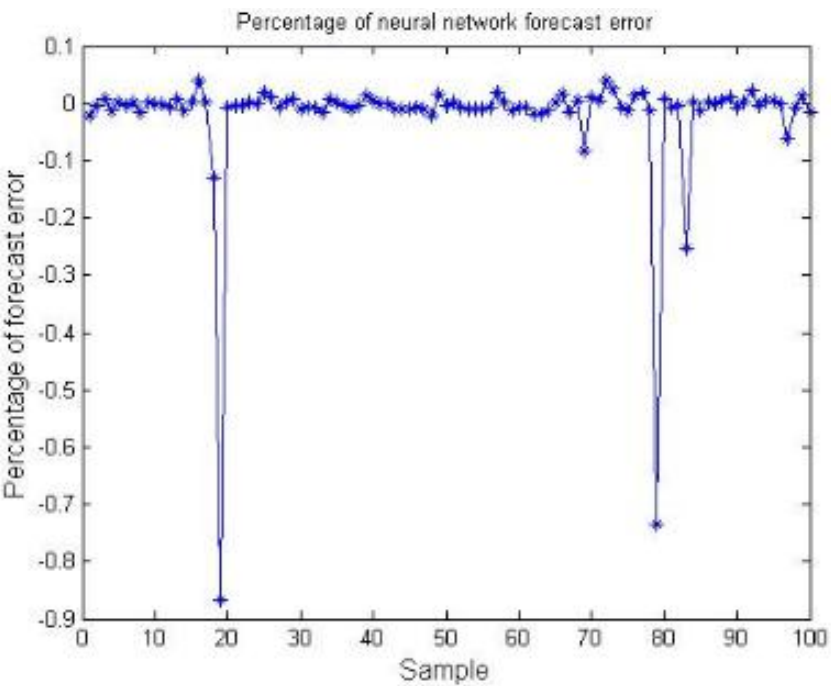

Fig. 4 Percentage of the forecast error

It can be seen from the diagram that the error control is about $10 \%$ which is in the acceptable range. So the BP neural network has good predictive power for the safety of gas stations.

Error improvement. By comparing the results of the error results, we found in individual the prediction deviation is big. This will cause false alarm of the risk analysis in the gas station. Therefore, it is necessary to improve the model. This paper compares t two kinds of error improved scheme: one is increasing the hidden layer number and the other one is changing the number of hidden layer neurons. 
When the hidden layer is added to two layers and the network structure is improved to 6-8-8-1, the output results are as figure 5.The percentage of the output error is about $2 \%$ compared with the single hidden layer. So the improvement of the system is very obvious when we appropriately increase the hidden layer in the appropriate amount of data.

When the number of hidden layer neurons is increased from 8 to 15 and the network structure is changed into 6-15-1, the output results are as shown in figure 6.It can be seen that the percentage of error is also the same as the number of neurons in the hidden layer is 8 , but the number of individual errors is increased.

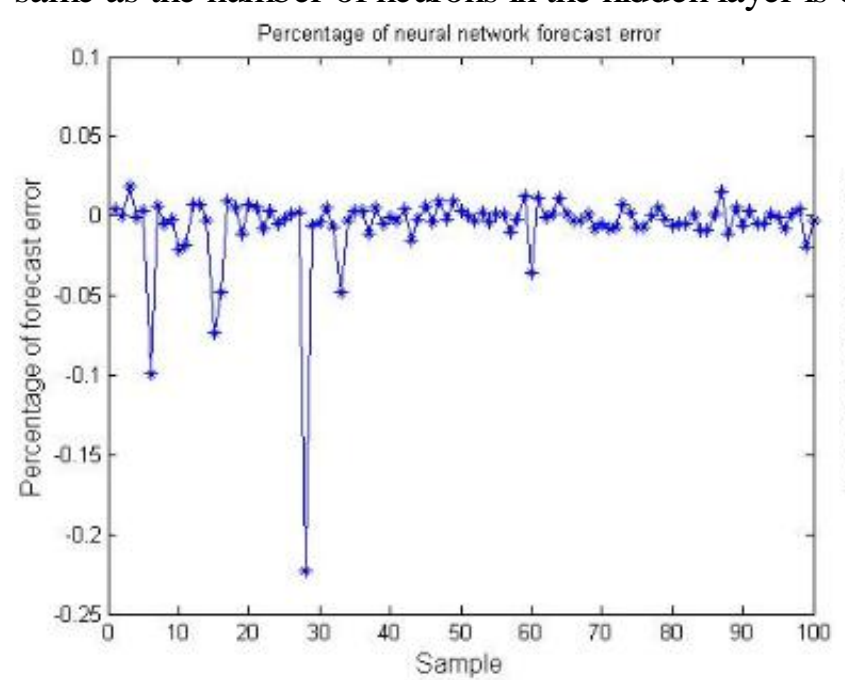

Fig. 5 double hidden layer error percentage

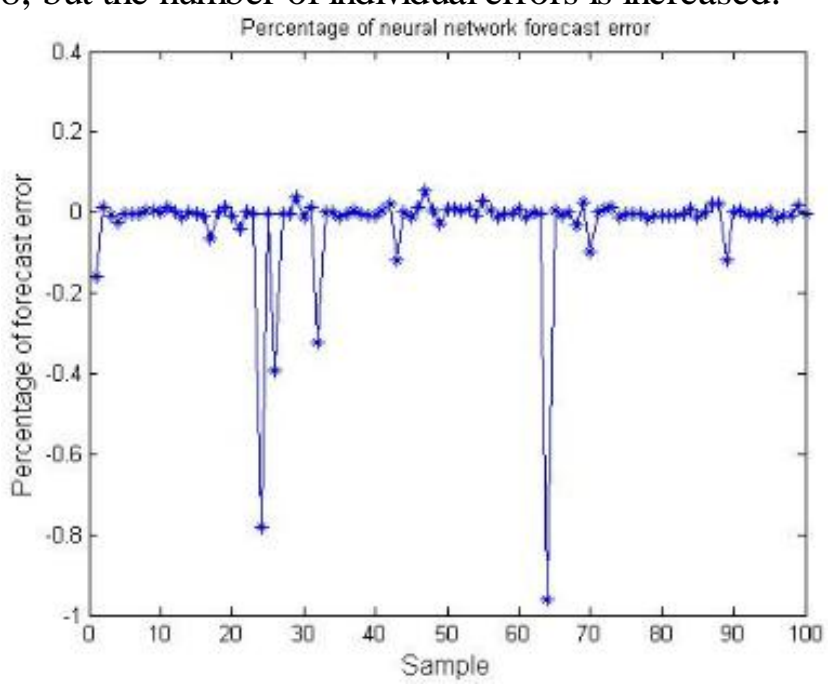

Fig. 6 neurons 15 error percentage

So the number of hidden layer neurons is not the more the better. The more number will make the network model become complex, reduce the network generalization ability, and increase the error. How to select the appropriate number of hidden layer neurons, we need to do more test to determine.

\section{Summary}

In this paper, the importance of safety monitoring of gas stations is discussed, and the BP neural network is used to predict the risk factors of gas stations. The results show that the method is feasible by modeling analysis, simulation and comparison of two improved schemes. However, as a kind of system application in large key monitoring sites, this paper only selects some data index in gas stations, but needs to be considered in practical application. Therefore, it is needed to collect different data.

\section{References}

[1] Wenzhong Guo. Discussion on the safety management of natural gas filling station [J]. Chemical Management, 2014, (23).

[2] Guo Yonghong. Analysis and Discussion on the running status of CNG gas station [J]. City Cas, 2007 (7): $11-15$.

[3] Xie Dianrong, Ni Xiaoyang, Guo Hailin, Shen Chi soldiers.Design of safety monitoring system for liquefied petroleum gas vehicle filling station.Safety and environmental engineering.2009 (9)

[4] Dong Changhong. Neural network and application M3. Beijing: National Defense Industry Press, 2005:202-204.

[5] Tang Shoufeng, Tong Minming, Dong Haibo. Sensor principle and application technology. Tsinghua University Press.2012.

[6] Lin Jun, A study of the convergence of neural networks in the back propagation. Computer and Modernization, 2005, (7): 34-36. 
[7] Kang Rongxue, Zhu Mingyu, Bao Junsheng and so on. Refueling station, safety monitoring and early warning system R \& D and application [J]. China Safety Science Journal Reported, 2008,18 (1): 33-35. (7): 156-160.

[8] Sang Haiquan,, Liu Ji, Wei Lijun et al. Research on dynamic supervision and emergency rescue platform for major hazard sources [J]. Chinese Journal of Safety Science, 2007,17 (7): 81-88.

[9] Wu Aiyou, Shi Shiliang, Wang Conglu. Urban fire risk assessment model based on neural network and genetic algorithm EJ3. China Safety Science Journal,, 2006,16 (3): 33-36. (11): 108 - 113.

[10] Chang Lin Su, Liu Jinlin, Qiu Dongli. Compressed natural gas (CNG filling station safety monitoring and information management [J]. China Measurement Technology, 2007,33 (5): 15-18.

[11] Wang Huaan. Safety monitoring of urban gas stations [J]. China Public Security (market version), 2007, (5) 142-143.

[12] Deng Bao, song Rui. Safety assessment method based on BP neural network EJ3. Security and Environment Engineering, 2005, 12 (2): 61 -64. 